\title{
Transgression and the Chern Character of Finite-Dimensional K-Cycles
}

\author{
Alain Connes ${ }^{1}$ and Henri Moscovici ${ }^{2 \star}$ \\ ${ }^{1}$ I.H.E.S., 91440 Bures-sur-Yvette, France \\ 2 Department of Mathematics, The Ohio State University, Columbus, OH 43210, USA
}

Received June 22, 1992; in revised form August 21, 1992

\section{Dedicated to Huzihiro Araki}

\begin{abstract}
It is shown that the [JLO] entire cocycle of a finitely summable unbounded Fredholm module can be retracted to a periodic cocycle. Moreover, the retracted cocycle admits a zero-temperature limit, which provides the extension of the transgressed cocycle of [CM1] from the invertible case to the general case.
\end{abstract}

\section{Introduction}

The Chern character theory of K-cycles over an algebra $A$, developed as an analogue of the classical index theory of elliptic differential operators on a closed smooth manifold $M$, plays a fundamental role in non-commutative geometry $([\mathrm{C} 1, \mathrm{C} 2])$. In this paper we are concerned with finite-dimensional K-cycles, i.e. with the K-cycles represented by unbounded finitely summable Fredholm modules over $A$.

Such a K-cycle $(\mathbf{H}, D)$ admits both a periodic Chern character, which is a class in the periodic cyclic cohomology $H C^{*}{ }_{\text {per }}(A)$, and an entire Chern character, belonging to the entire cyclic cohomology $H C^{*}{ }_{\text {ent }}(A)$. The periodic cyclic cohomology is much better understood than the entire cohomology, and is explicitly computed for many interesting algebras. On the other hand, the Jaffe-LesniewskiOsterwalder cocycle [JLO], representing the entire Chern character (cf. [C3]), has some computational advantages over the periodic cocycle.

This tension can be detected already in the case when $A=C^{\infty}(M)$, with $M$ a spin manifold, and $D=$ the Dirac operator on $M$. Indeed, it is then known (cf. $\left[\mathrm{C} 1\right.$, Part II, §6]) that $H C^{\mathrm{ev}}{ }_{\text {per }}\left(C^{\infty}(M)\right) \cong H_{\mathrm{ev}}{ }^{\mathrm{dR}}(M, \mathbb{C})$, resp. $H C^{\text {odd }}{ }_{\text {per }}\left(C^{\infty}(M)\right) \cong$ $H_{\text {odd }}{ }^{\mathrm{dR}}(M, \mathbb{C})$, whereas the similar isomorphism for $H C^{*}{ }_{\text {ent }}\left(C^{\infty}(M)\right)$, expected to hold as well, was proved so far only for $M=\mathbb{S}^{1}$. By contrast, it is relatively easier to recover the $\hat{A}$-class of the manifold $M$ from the entire JLO cocycle (cf. $[\mathrm{BF}]$ ) than from the periodic cocycle (cf. [C1, Part I, Thm. 6.5]).

^ Research supported in part by NSF Grant DMS-9101557 
In this article we resolve the above issue by producing a formula which preserves the advantages of the JLO cocycle but still represents the periodic Chern character. This formula is obtained by means of a "transgressive" retraction of the entire JLO cocycle to a periodic cocycle. A remarkable feature of the "retracted" cocycle is that it admits a "zero-temperature" limit, which coincides with the transgressed cocycle constructed in [CM1] when $D$ is invertible.

A brief outline of the paper is now in order. The basic definitions are recalled in Sect. 1. Section 2 begins with the retraction of the JLO cocycle to a periodic cocycle. We then prove the existence of the zero-temperature limit for the retracted cocycle, thus extending the construction of the transgressed cocycle of [CM1] to the general case. The "correction" needed for this extension is provided by the zero-temperature limit of the components of the JLO cocycle, which in turn is given by cochains reminiscent of the cocycles associated to algebra extensions ( $[\mathrm{C} 1$, Part $\mathrm{I}, \S 3],[Q])^{1}$.

The fact that the cocycles constructed in Sect. 2 represent precisely the same periodic Chern character as the one originally defined in [C1], is proved in Sect. 3. In the same section we give the corresponding formula for the index pairing.

Section 4 deals with the "infinite-temperature" limit, which may no longer exist in general. We show that if one postulates the existence of large temperature asymptotic expansions for the components of the JLO cocycle and their transgressed versions - modelled after the case of an elliptic pseudo-differential operator on a closed manifold - the Chern character can be represented by a "local" formula, i.e. by an expression which is a direct generalization of the current representing the index class of an elliptic operator. In particular, one recovers the $\hat{A}$-class of a spin manifold $M$ as precisely the periodic Chern Character of the Dirac operator. We end with a non-commutative illustration of the present formalism, by identifying explicitly the Chern characters of K-cycles over the Toeplitz algebra of the circle.

\section{Cyclic Cohomology and the Chern Character}

For the convenience of the reader, and also to set the notation, we recall in this section some basic definitions.

1.1. The Cyclic Complex. (cf. [C1, Part II, §1]). Given a Banach algebra with unit $A$ over $\mathbb{C}$, let

$$
\begin{aligned}
& C^{n}(A)=\text { the space of continuous }(n+1) \text {-linear forms on } A \text {, if } n \geqq 0 ; \\
& C^{n}(A)=0 \text {, if } n<0 .
\end{aligned}
$$

Furthermore, let

$$
\begin{gathered}
C_{\lambda}{ }^{n}(A)=\text { the subspace of } C^{n}(A) \text { satisfying the cyclicity condition } \\
\qquad \phi^{n}\left(a_{n}, a_{0}, \ldots, a_{n-1}\right)=(-1)^{n} \phi^{n}\left(a_{0}, \ldots, a_{n}\right) .
\end{gathered}
$$

\footnotetext{
${ }^{1}$ Another interesting analogy, with the treatment in [BGV, Chap. 10] of the local family index theorem, was pointed out to us by Ezra Getzler
} 
The Hochschild coboundary operator $b: C^{n-1}(A) \rightarrow C^{n}(A)$,

$$
\begin{aligned}
b \phi^{n-1}\left(a_{0}, \ldots, a_{n}\right)= & \sum_{0 \leqq j \leqq n-1}(-1)^{j} \phi^{n-1}\left(a_{0}, \ldots, a_{j} a_{j+1}, \ldots, a_{n}\right) \\
& +(-1)^{n} \phi^{n-1}\left(a_{n} a_{0}, \ldots, a_{n-1}\right)
\end{aligned}
$$

preserves the invariant subspaces of the cyclic permutator, so that

$$
\mathbf{C}_{\lambda}(A)=\left(C_{\lambda}{ }^{n}(A), b\right)
$$

is itself a complex. Its homology groups $H_{\lambda}{ }^{n}(A)$ form the cyclic cohomology of $A$.

1.2. The (b,B)-Bicomplex. (cf. [C1, Part II, §4], also [LQ]). Let $C^{n, m}(A)=$ $C^{n-m}(A)$. The Hochschild operator defines horizontal differentials b: $C^{n-1, m}(A) \rightarrow C^{n, m}(A)$, while the operator $B: C^{n+1}(A) \rightarrow C^{n}(A)$,

$$
\begin{aligned}
B \phi^{n+1}\left(a_{0}, \ldots, a_{n}\right)= & \sum_{0 \leqq j \leqq n}(-1)^{n j}\left(\phi^{n+1}\left(1, a_{n-j+1}, \ldots, a_{n}, a_{0}, \ldots, a_{n-j}\right)\right. \\
& \left.+(-1)^{n} \phi^{n+1}\left(a_{n-j+1}, \ldots, a_{n}, a_{0}, \ldots, a_{n-j}, 1\right)\right),
\end{aligned}
$$

defines vertical differentials $B: C^{n, m}(A) \rightarrow C^{n, m+1}(A)$. One has

$$
b^{2}=B^{2}=b B+B b=0,
$$

therefore $\mathbf{C}(A)=\left(C^{n, m}(A), b\right), B$ is a bicomplex.

Consider now the "first quadrant" subcomplex $\mathbf{C}_{+}(A)=\left(C^{n, m}(A), b, B\right)_{n \geqq 0, m \geqq 0}$. It can be shown that the inclusion of the cyclic complex $\mathbf{C}_{\lambda}(A)$ into the total complex $\mathrm{Tot}_{+}(A)$ of $\mathbf{C}_{+}(A)$ is a quasi-isomorphism. Therefore, it induces an isomorphism between $H_{\lambda}{ }^{n}(A)$ and the corresponding homology groups $\operatorname{HC}^{n}(A)$. There is an obvious shift identifying $C^{n, m}(A)$ with $C^{n+1, m+1}(A)$, which defines an embedding of degree two of the total complex into itself. This, in turn, induces on cyclic cohomology the periodicity operator $S: H C^{n}(A) \rightarrow H C^{n+2}(A)$.

For the full bicomplex $\mathbf{C}(A)$ there are two ways of forming a total complex, depending on whether the $n$-cochains are defined as the direct sum or the direct product of $C^{p, q}(A)$, with $p+q=n$. If one uses the direct product, the resulting total complex $\operatorname{Tot}_{\Pi}(A)$ of "infinite" cochains has trivial homology: $H C_{\text {inf }}{ }^{n}(A)=0$. If one uses the direct sum, the resulting total complex $\operatorname{Tot}_{\Sigma}(A)$ of "finite" cochains gives the periodic cyclic cohomology $H C_{\text {per }}{ }^{n}(A)$. The "periodicity" shift mentioned above shows that, in effect, there are only two kinds of periodic cyclic cohomology groups: even and odd.

1.3. The Entire Complex. (cf. [C2]). In order to obtain an interesting cohomology out of the total complex of infinite cochains, one needs to restrict the "growth" of these cochains. This can be achieved by considering the subcomplex $\mathbf{C}_{\text {ent }}(A)$ of entire cochains, i.e. of those cochains $\left\{\phi^{n}\right\}_{n \geqq 0}$ such that the series $\sum_{n \geqq 0}(n !)^{1 / 2}\left\|\phi^{n}\right\| z^{n}$ defines an entire function of $z$. It is a $Z_{2}$-graded complex, whose cohomology $H C_{\text {ent }}{ }^{ \pm}(A)$ is called the entire cohomology of $A$.

1.4. Fredholm Modules. (cf. [C1, C2]). An odd (ungraded) unbounded p-summable Fredholm module over $A$ consists of a Hilbert space $\mathbf{H}$ equipped with a continuous 
representation $A \rightarrow \mathscr{L}(\mathbf{H})$, together with a densely defined unbounded self-adjoint operator $D$ on $\mathbf{H}$, satisfying the following properties:

(i) $\exists p \in[1, \infty)$ such that $\operatorname{Tr}\left(I+D^{2}\right)^{-p / 2}<\infty$;

(ii) $\forall a \in A$ the operator $[D, a]$ (is densely defined and) extends to a bounded operator on $\mathbf{H}$; for future use, we note that $\exists C_{D}<\infty$ such that

$$
\|a\|+\|[D, a]\| \leqq C_{D}\|a\|_{A} .
$$

An even $\left(Z_{2}\right.$-graded $)$ unbounded, p-summable Fredholm module over $A$ is defined by the same data with the addition of a self-adjoint involution $\gamma \in \mathscr{L}(\mathbf{H})$ satisfying:

(iii) $D \gamma=-\gamma D$ and $a \gamma=\gamma a, \forall a \in A$.

Replacing the $p$-summability condition (i) by the weaker, $\vartheta$-summability requirement

(i') $\operatorname{Tr} e^{-t D^{2}}<\infty, \forall t>0$,

one obtains the notion of $\vartheta$-summable Fredholm module.

1.5. The (Periodic) Chern Character. (cf. $[\mathrm{C} 1])$. If $(\mathbf{H}, D)$ is an even $p$-summable Fredholm module with the property that $D$ is invertible, its $n^{\text {th }}$ Chern character is defined as the class

$$
\operatorname{ch}^{n}(\mathbf{H}, D)=\left[{\phi_{D}}^{n}\right] \in H C^{n}(A), \text { for } n=2 k \geqq p-1,
$$

of the cyclic cocycle

$$
\phi_{D}{ }^{n}\left(a_{0}, \ldots, a_{n}\right)=c_{n} \operatorname{Str}\left(D^{-1}\left[D, a_{0}\right] \ldots D^{-1}\left[D, a_{n}\right]\right),
$$

where $c_{n}$ are suitable normalizing constants, depending only on $n$.

One has

$$
\operatorname{Sch}^{n}(\mathbf{H}, D)=\operatorname{ch}^{n+2}(\mathbf{H}, D),
$$

which makes possible to define, independently of $n$, the periodic Chern character of (H, D):

$$
\operatorname{ch}(\mathbf{H}, D)=\left[\phi_{D}{ }^{n}\right]_{\mathrm{per}} \in H C_{\mathrm{per}}^{\mathrm{ev}}(A) .
$$

Let us also recall that in [CM1] we constructed, by a process of transgression, another cocycle representing the same Chern character, namely:

$$
\begin{gathered}
\tau_{D}{ }^{n}\left(a_{0}, \ldots, a_{n}\right)=\sum_{\lambda} \operatorname{sgn}(\lambda) \int_{0}^{\infty} d t t^{n+1} \int_{\Delta_{n+1}} \operatorname{Str}\left(D e^{-t_{0} t^{2} D^{2}}\left[D, a_{\lambda(0)}\right] e^{-\left(t_{1}-t_{0}\right) t^{2} D^{2}} \ldots\right. \\
\left.\ldots\left[D, a_{\lambda(n)}\right] e^{-\left(1-t_{n}\right) t^{2} D^{2}}\right) d t_{0} \ldots d t_{n}, \quad n=2 k>p-1,
\end{gathered}
$$

where $\lambda$ runs through the cyclic permutations of $\{0,1, \ldots, n\}$ and $\Delta_{n+1}$ is the standard $(n+1)$-dimensional simplex.

These definitions can be extended to the general case in the following canonical fashion. Let $(\mathbf{h}, F)$ be the graded 0 -summable Fredholm module defined by

$$
\mathbf{h}^{+}=\mathbb{C}, \quad \mathbf{h}^{-}=\mathbb{C}, \quad F=\left[\begin{array}{ll}
0 & 1 \\
1 & 0
\end{array}\right],
$$


the module structure over $\mathbb{C}$ being that for which $1 \in \mathbb{C}$ act on $\mathbf{h}$ as $\left[\begin{array}{ll}1 & 0 \\ 0 & 0\end{array}\right]$. Form the graded tensor product $\mathbf{H} \otimes_{\mathrm{gr}} \mathbf{h}$, viewed as an $A=A \otimes \mathbb{C}$-module, and consider the operator $D_{m}=D \otimes_{\mathrm{gr}} I+m I \otimes_{\mathrm{gr}} F, m \in \mathbb{R}$. For $m \neq 0,\left(\mathbf{H}, \otimes_{\mathrm{gr}} \mathbf{h}, D_{m}\right)$ is an invertible $p$-summable unbounded Fredholm module, and its Chern character $\operatorname{ch}^{n}\left(\mathbf{H} \otimes_{\mathrm{gr}} \mathbf{h}, D_{m}\right) \in H C_{\mathrm{per}}^{\mathrm{ev}}(A), n=2 k \geqq p-1$, is independent of $m$ (cf. [C1, Part I, Prop. 6.4]).

Furthermore, the odd case can be reduced to the even one by a standard procedure: first, one extends the above definition, in a straightforward way, to the case of graded algebras; then, one replaces the ungraded Fredholm module $(\mathbf{H}, D)$ by a canonically associated $Z_{2}$-graded Fredholm module over $A \otimes_{\mathrm{gr}} \mathbf{C}_{1}, \mathbf{C}_{1}$ being the complex Clifford algebra over the 1-dimensional Euclidean space. (See [C1, Part I, §7].)

1.6. The Entire Chern Character. (cf. [C2], also [JLO]). The Chern character of a $\vartheta$-summable Fredholm module $(\mathbf{H}, D)$ was defined in $[C 2]$, as an entire cyclic class in $H C_{\text {ent }}{ }^{ \pm}(A)$. We shall need here the equivalent (cf. [C3]) but more convenient [JLO] version, which we proceed now to recall.

Let $\Delta_{n}=\left\{\left(t_{1}, \ldots, t_{n}\right) \in \mathbb{R}^{n} ; 0 \leqq t_{1} \leqq \ldots \leqq t_{n} \leqq 1\right\}$. Given $A_{0}, \ldots, A_{n} \in$ $\mathscr{L}(\mathbf{H})$, we set

$$
\left\langle A_{0}, \ldots, A_{n}\right\rangle_{D}=\int_{\Delta_{n}} \operatorname{Tr}\left(\gamma A_{0} e^{-t_{1} D^{2}} A_{1} e^{-\left(t_{2}-t_{1}\right) D^{2}} \ldots A_{n} e^{-\left(1-t_{n}\right) D^{2}}\right) d t_{1} \ldots d t_{n},
$$

with the convention $\gamma=I$ (the identity operator) if $(\mathbf{H}, D)$ is ungraded. In this notation, the [JLO] cocycle representing the entire Chern character of $(\mathbf{H}, D)$ is given by the following components:

$$
\operatorname{Ch}^{n}(D)\left(a_{0}, \ldots, a_{n}\right)=\left\langle a_{0},\left[D, a_{1}\right], \ldots,\left[D, a_{n}\right]\right\rangle_{D}, a_{0}, \ldots, a_{n} \in A,
$$

where $n$ runs through all positive integers of the same parity as $(\mathbf{H}, D)$. The fact that it gives a cocycle is expressed by the identity

$$
b \mathrm{Ch}^{n-1}(D)+B \mathrm{Ch}^{n+1}(D)=0 .
$$

In addition, it satisfies the transgression formula

$$
-d / d t \mathrm{Ch}^{n}(t D)=b \phi \mathrm{h}^{n-1}(t D, D)+B \notin \mathrm{h}^{n+1}(t D, D),
$$

where, for an operator $V$ on $\mathbf{H}$ of degree $[V] \in Z_{2}$, we have denoted

$$
\begin{aligned}
& \phi \mathrm{h}^{n}(D, V)\left(a_{0}, \ldots, a_{n}\right) \\
& \quad=\sum_{0 \leqq i \leqq n}(-1)^{i[V]}\left\langle a_{0},\left[D, a_{1}\right], \ldots,\left[D, a_{i}\right], V,\left[D, a_{i+1}\right], \ldots,\left[D, a_{n}\right]\right\rangle_{D} .
\end{aligned}
$$

\section{Conversion of the JLO Cocycle}

In this section $(\mathbf{H}, D)$ will denote an arbitrary unbounded $p$-summable Fredholm module over $A$. 
2.1. The Retracted Cocycle. We shall show here that the JLO entire cocycle can be converted into a periodic cyclic cocycle, by replacing its tail with a finite-length transgression. First, we need some simple estimates which follow from the $p$ summability assumption.

Lemma 1. Let $n>p$. Then

(a) $\lim _{t \rightarrow 0} \mathrm{Ch}^{n}(t D)=0$;

(b) the function $t \rightarrow\left\|\phi \mathrm{h}^{n}(t D, D)\right\|$ is integrable on $[0, T], \forall T<\infty$.

Proof. (a) The Hölder inequality for operators gives

$$
\left\|\mathrm{Ch}^{n}(t D)\right\| \leqq(n) !^{-1} C_{D}{ }^{n+1} t^{n} \operatorname{Tr}\left(e^{-t^{2} D^{2}}\right) .
$$

On the other hand, since

$$
\begin{aligned}
\operatorname{Tr} e^{-t^{2} D^{2}} & =\operatorname{Tr}\left(\left(I+D^{2}\right)^{p / 2} e^{-t^{2} D^{2}}\left(I+D^{2}\right)^{-p / 2}\right) \\
& \leqq(p / 2 e)^{p / 2} t^{-p} e^{t^{2}} \operatorname{Tr}\left(I+D^{2}\right)^{-p / 2}
\end{aligned}
$$

one sees that

$$
\operatorname{Tr} e^{-t^{2} D^{2}}=O\left(t^{-p}\right) \quad \text { as } t \rightarrow 0^{+} .
$$

(b) A more judicious use of Hölder's inequality (see [GS, Lemma 2.1]), gives

$$
\left\|\notin \mathrm{h}^{n}(t D, D)\right\| \leqq(n) !^{-1}(n+1) C_{D}{ }^{n} \delta^{-1 / 2} t^{n-1} \operatorname{Tr}\left(e^{-(1-\delta) t^{2} D^{2}}\right), \quad \forall 0<\delta<1 .
$$

Therefore,

$$
\left\|\phi \mathrm{h}^{n}(t D, D)\right\|=O\left(t^{n-p-1}\right) \text { as } t \rightarrow 0^{+},
$$

which ensures integrability on $[0, T]$.

In view of (b) above, we can define for $\forall n>p$ and $t<\infty$ a continuous $(n+1)$-linear form on $A$ by setting

$$
\mathrm{T}_{\phi} \mathrm{h}_{t}{ }^{n}(D)\left(a_{0}, \ldots, a_{n}\right)=\int_{0}^{t} \phi \mathrm{h}^{n}(s D, D)\left(a_{0}, \ldots, a_{n}\right) d s .
$$

Proposition 1. For any $n>p-1, n$ of the same parity as $(\mathbf{H}, D)$, and any $t>0$, the formula

$$
\operatorname{ch}_{t}{ }^{n}(D)=\sum_{k \geqq 0} \mathrm{Ch}^{n-2 k}(t D)+B \mathrm{~T} \phi \mathrm{h}_{t}{ }^{n+1}(D)
$$

defines a cocycle in Tot ${ }_{+}(A)$; its cohomology class in $H C^{n}(A)$ is independent of $t>0$. Moreover, $S\left(\operatorname{ch}_{t}^{n}(D)\right)$ and $\mathrm{ch}_{t}{ }^{n+2}(D)$ are cohomologous in $\operatorname{Tot}_{\Sigma}(A)$; in particular, the corresponding periodic cyclic cohomology class is independent of $n$.

Proof. Integrating the transgression formula (1.2) from $\varepsilon>0$ to $t>0$ gives

$$
\mathrm{Ch}^{n}(\varepsilon D)-\mathrm{Ch}^{n}(t D)=b \int_{\varepsilon}^{t} \phi \mathrm{h}^{n-1}(s D, D) d s+B \int_{\varepsilon}^{t} \phi \mathrm{h}^{n+1}(s D, D) d s .
$$


Therefore, using the cocycle property (1.1),

$$
b\left(\mathrm{Ch}^{n}(t D)+B \int_{\varepsilon}^{t} \phi \mathrm{h}^{n-1}(s D, D) d s\right)=b \mathrm{Ch}^{n}(\varepsilon D)=-B \mathrm{Ch}^{n+2}(\varepsilon D) .
$$

In view of Lemma 1 , we can let $\varepsilon \rightarrow 0$ to obtain

$$
b\left(\mathrm{Ch}^{n}(t D)+B \mathrm{~T} \notin D h_{t}{ }^{n+1}(D)\right)=0 .
$$

It follows that

$$
\begin{aligned}
-B \operatorname{ch}_{t}{ }^{n}(D) & =-\sum_{k \geqq 0} B \mathrm{Ch}^{n-2 k}(t D)=\sum_{k \geqq 0} b \mathrm{Ch}^{n-2 k-2}(t D) \\
& =\sum_{k \geqq 0} b \mathrm{Ch}^{n-2 k-2}(t D)+b\left(\mathrm{Ch}^{n}(t D)+B \mathrm{~T}_{\phi} \mathrm{h}_{t}{ }^{n+1}(D)\right) \\
& =b \phi \mathrm{h}_{t}{ }^{n}(D),
\end{aligned}
$$

i.e. $\operatorname{ch}_{t}{ }^{n}(D)$ is a cocycle.

Furthermore,

$$
\begin{aligned}
\operatorname{ch}_{t}{ }^{n}(D)-\operatorname{ch}_{\tau}{ }^{n}(D) & =\sum_{k \geqq 0}\left(\mathrm{Ch}^{n-2 k}(t D)-\mathrm{Ch}^{n-2 k}(\tau D)\right)+B \int_{\tau}^{t} \phi \mathrm{h}^{n+1}(s D, D) d s \\
& =-(b+B) \sum_{k \geqq 0} \int_{\tau}^{t} \phi \mathrm{h}^{n-2 k-1}(s D, D) d s .
\end{aligned}
$$

To prove the second assertion we note that, after replacing $n$ by $n+2$ in (2.1), we can let $\varepsilon \rightarrow 0$ to get, again by Lemma 1 ,

$$
\mathrm{Ch}^{n+2}(t D)=-b \mathrm{~T} \phi \mathrm{h}_{t}{ }^{n+1}(D)-B \mathrm{~T} \phi \mathrm{h}_{t}{ }^{n+3}(D) \text {. }
$$

Hence,

$$
\begin{aligned}
\operatorname{ch}_{t}{ }^{n+2}(D)-\operatorname{ch}_{t}{ }^{n}(D)= & \operatorname{Ch}^{n+2}(t D)+B \mathrm{~T} \phi \mathrm{h}_{t}{ }^{n+3}(D)-B \mathrm{~T} \phi \mathrm{h}_{t}{ }^{n+1}(D) \\
& =-(b+B) \mathrm{T} \phi \mathrm{h}^{n+1}(D) .
\end{aligned}
$$

2.2. The Zero-temperature Limit. In order to relate the above cyclic cocycle to the transgressed cocycle of [CM1], we need to prove existence of the "zero-temperature" limit. This will be a consequence of the following result.

Lemma 2. For any $n \geqq 1$ and $a_{0}, \ldots, a_{n} \in A$,

$$
\mathrm{ch}^{n}(t D, D)\left(a_{0}, \ldots, a_{n}\right)=O\left(t^{-2}\right) \quad \text { as } t \rightarrow \infty ;
$$

if $n$ is odd, one has in fact

$$
\phi \mathrm{h}^{n}(t D, D)\left(a_{0}, \ldots, a_{n}\right)=O\left(t^{-3}\right) \quad \text { as } t \rightarrow \infty
$$

Proof. We recall that $\phi \mathrm{h}^{n}(t D, D)\left(a_{0}, \ldots, a_{n}\right)=\sum_{0 \leqq i \leqq n}(-1)^{i} T_{i}$, where

$$
\begin{aligned}
T_{i}= & \left\langle a_{0},\left[t D, a_{1}\right], \ldots,\left[t D, a_{i}\right], D,\left[t D, a_{i+1}\right], \ldots,\left[t D, a_{n}\right]\right\rangle_{t D} \\
= & t^{n} \int_{\Delta_{n+1}} \operatorname{Tr}\left(\gamma a_{0} e^{-t_{1} t^{2} D^{2}}\left[D, a_{1}\right] e^{-\left(t_{2}-t_{1}\right) t^{2} D^{2}} \ldots\right. \\
& {\left[D, a_{i}\right] e^{-\left(s-t_{i}\right) t^{2} D^{2}} D e^{-\left(t_{t}+1-s\right) t^{2} D^{2}}\left[D, a_{i+1}\right] } \\
& \left.\ldots\left[D, a_{n}\right] e^{-\left(1-t_{n}\right) t^{2} D^{2}}\right) d t_{1} \ldots d s \ldots d t_{n} .
\end{aligned}
$$


Integrating with respect to $d s, t_{i} \leqq s \leqq t_{i+1}, T_{i}$ takes the form

$$
\begin{aligned}
T_{i}= & t^{n} \int_{\Delta_{n}}\left(t_{i+1}-t_{i}\right) \operatorname{Tr}\left(\gamma a_{0} e^{-t_{1} t^{2} D^{2}}\left[D, a_{1}\right] e^{-\left(t_{2}-t_{1}\right) t^{2} D^{2}} \ldots\right. \\
& {\left.\left[D, a_{i}\right] D e^{-\left(t_{i+1}-t_{i}\right) t^{2} D^{2}}\left[D, a_{i+1}\right] \ldots\left[D, a_{n}\right] e^{-\left(1-t_{n}\right) t^{2} D^{2}}\right) d t_{1} \ldots d t_{n} . }
\end{aligned}
$$

It will be convenient to reparametrize the $n$-simplex by the coordinates

$$
s_{0}=t_{1}, \ldots, s_{j}=t_{j+1}-t_{j}, \ldots, s_{n}=1-t_{n},
$$

and thus regard it as the set $\left\{\left(s_{0}, s_{1}, \ldots, s_{n}\right) \in\left(\mathbb{R}^{+}\right)^{n+1} ; s_{0}+s_{1}+\cdots+s_{n}=1\right\}$, but still equipped with the Lebesgue measure $d_{n} \sigma=d t_{1} \ldots d t_{n}$. Then

$$
\begin{aligned}
T_{i}= & t^{n} \int_{\Delta_{n}} s_{i} \operatorname{Tr}\left(\gamma a_{0} e^{-s_{0} t^{2} D^{2}}\left[D, a_{1}\right] e^{-s_{1} t^{2} D^{2}} \ldots\right. \\
& {\left.\left[D, a_{i}\right] D e^{-s_{i} t^{2} D^{2}}\left[D, a_{i+1}\right] \ldots\left[D, a_{n}\right] e^{-s_{n} t^{2} D^{2}}\right) d_{n} \sigma . }
\end{aligned}
$$

We now split $T_{i}$ into a sum, by decomposing each heat operator into components corresponding to the orthogonal projection $H$ onto $\operatorname{Ker} D$ and to its complement:

$$
e^{-s_{J} t^{2} D^{2}}=H+e^{-s_{J} t^{2} D^{2}}(I-H) .
$$

Since $H\left[D, a_{j}\right] H=0$, only the terms containing no more than $[n / 2]+1$ copies of $H$ can give a non-zero contribution. On the other hand, the term containing no copy of $H$ at all corresponds to the case of an invertible operator, which was treated in $[\mathrm{CM} 1, \S 6]$ and was shown to have exponential decay.

Thus, we are left to treat the terms containing at least [n/2] (resp., when $n$ is odd, $[n / 2]+1)$ copies of $I-H$, as well as at least one copy of $H$. Consider such a term, $T_{i ; j_{0}, j_{1}, \ldots, j_{q}}$, where $j_{0}$ is the index corresponding to one of the copies of $H$ and $j_{1}, \ldots, j_{q}$ are the indices corresponding to those heat kernels which are followed by $I-H$; note that $i$ is necessarily one of the latter, say $j_{h}$. Thus, $T_{i ; j_{0}, j_{1}, \ldots, j_{q}}$ is of the form

$$
\begin{aligned}
T_{i ; j_{0}, j_{1}, \ldots, j_{q}}= & t^{n} \int_{\Delta_{n}} s_{j_{h}} \operatorname{Tr}\left(\gamma \ldots e^{-s_{J_{k}} t^{2} D^{2}}(I-H)\right. \\
& \left.\ldots H \ldots\left[D, a_{j_{h}}\right] D e^{-s_{j_{h}} t^{2} D^{2}}(I-H) \ldots\right) d_{n} \sigma,
\end{aligned}
$$

where $1 \leqq k \leqq q$. In particular, the variables $s_{j}$ with $j \neq j_{0}, j_{1}, \ldots, j_{q}$, as well as $s_{j_{0}}$, do not appear in the integrand. Also, all the remaining heat operators correspond to the invertible part of $D$.

It will be convenient to reparametrize once more $\Delta_{n}$ by identifying it with its projection along the $s_{j_{0}}$-axis, i.e. viewing it as the set

$$
\left\{\left(s_{0}, s_{1}, \ldots, s_{j_{0}-1}, s_{j_{0}+1}, \ldots, s_{n}\right) \in\left(\mathbb{R}^{+}\right)^{n} ; \sum_{j \neq j_{0}} s_{j} \leqq 1\right\},
$$

and then perform the substitution

$$
u_{k}=t^{2} s_{j_{k}} \text { for } k=1, \ldots, q \text { and } v_{j}=s_{j} \text { for } j \neq j_{0}, j_{1}, \ldots, j_{q} .
$$

If one integrates first in the variables $v_{j}$, over the simplex

$$
\sum_{j \neq j_{0}, j_{1}, \ldots, j_{q}} v_{j} \leqq 1-t^{-2}\left(u_{1}+\cdots+u_{q}\right),
$$


and then one integrates over

$$
\Delta_{q}(t)=\left\{\left(u_{1}, \ldots, u_{q}\right) \in\left(\mathbb{R}^{+}\right)^{q} ; u_{1}+\cdots+u_{q} \leqq t^{2}\right\},
$$

one obtains

$$
\begin{aligned}
T_{i ; j_{0}, j_{1}, \ldots, j_{q}}= & (n-q) !^{-1} t^{n-2 q-2} \int_{\Delta_{q(t)}} u_{h}\left(1-t^{-2}\left(u_{1}+\cdots+u_{q}\right)\right)^{n-q} \\
& \times \operatorname{Tr}\left(\gamma \ldots e^{-u_{k} D^{2}}(I-H) \ldots H \ldots\left[D, a_{j_{h}}\right]\right. \\
& \left.\times D e^{-u_{h} D^{2}}(I-H) \ldots\right) d_{n} \sigma .
\end{aligned}
$$

Remark that when $t \rightarrow \infty$ the domain of integration becomes $\left(\mathbb{R}^{+}\right)^{q}$, with the standard Lebesgue measure and the integral is dominated by

$$
\begin{gathered}
\int_{\left(R^{+}\right)_{q}} u_{h}\left|1+\left(u_{1}+\cdots+u_{q}\right)\right|^{n-q} \operatorname{Tr}\left(\gamma \ldots e^{-u_{k} D^{2}}(I-H) \ldots\right. \\
\left.\ldots H \ldots\left[D, a_{j_{h}}\right] D e^{-u_{h} D^{2}}(I-H) \ldots\right) \mid d u_{1} \ldots d u_{q}<\infty .
\end{gathered}
$$

So, to complete the proof it only remains to note that, since $q \geqq[n / 2]$ (resp. $[n / 2]+1$ for $n$ odd), the exponent of the outside power of $t$ is at most -2 (resp. -3 ).

As a consequence, it follows, using the identity (1.3), that the limit

$$
\mathrm{Ch}^{n}(\infty D)=\lim _{t \rightarrow \infty} \mathrm{Ch}^{n}(t D)
$$

exists for any $n$. We shall now compute it explicitly. Let $\rho_{H}$ denote the compression of $A$ to $\operatorname{Ker} D$, i.e.

$$
\rho_{H}(a)=H a H, \quad \forall a \in A,
$$

where $H=$ the orthogonal projection on $\operatorname{Ker} D$, and let

$$
\omega_{H}(a, b)=\rho_{H}(a b)-\rho_{H}(a) \rho_{H}(b), \quad a, b \in A
$$

be the corresponding curvature (see [Q]).

Proposition 2. (a) If $n$ is odd then $\mathrm{Ch}^{n}(\infty \mathrm{D})=0$.

(b) If $n=2 k$ then $\forall a_{0}, \ldots, a_{2 k} \in A$,

$$
\mathrm{Ch}^{2 k}(\infty D)\left(a_{0}, \ldots, a_{2 k}\right)=(-1)^{k} k !^{-1} \operatorname{Str}\left(\rho_{H}\left(a_{0}\right) \omega_{H}\left(a_{1}, a_{2}\right) \ldots \omega_{H}\left(a_{2 k-1}, a_{2 k}\right)\right) \text {. }
$$

Proof. By definition,

$$
\begin{aligned}
& \mathrm{Ch}^{n}(t D)\left(a_{0}, \ldots, a_{n}\right)=\left\langle a_{0},\left[t D, a_{1}\right], \ldots,\left[t D, a_{n}\right]\right\rangle_{t D} \\
&\left.\quad=t^{n} \int_{\Delta_{n}} \operatorname{Tr}\left(\gamma a_{0} e^{-t_{1} t^{2} D^{2}}\left[D, a_{1}\right] e^{-\left(t_{2}-t_{1}\right.}\right)^{t^{2} D^{2}} \ldots\left[D, a_{n}\right] e^{-\left(1-t_{n}\right) t^{2} D^{2}}\right) d_{n} \sigma \\
&=t^{n} \int_{\Delta_{n}} \operatorname{Tr}\left(\gamma a_{0} e^{-s_{0} t^{2} D^{2}}\left[D, a_{1}\right] e^{-s_{1} t^{2} D^{2}} \ldots\left[D, a_{n}\right] e^{-s_{n} t^{2} D^{2}}\right) d_{n} \sigma .
\end{aligned}
$$

As above, we shall split the integral into a sum of terms obtained by decomposing each heat operator according to the complementary orthogonal projections $H$ and $I-H$. Again as above, only the terms containing no more than $[n / 2]+1$ copies of $H$ may contribute nontrivially. On the other hand, using the Hölder inequality, one 
can easily see that the term containing no copy of $H$ decreases exponentially. Thus, as before, we are left to handle only the terms containing at least one copy of $H$ and at least $[n / 2]$ (resp. [n/2] +1 , if $n$ is odd) copies of $I-H$.

Let $S_{j_{0}, j_{1}, \ldots, j_{q}}$ be such a term, with $j_{0}$ corresponding to one of the copies of $H$ and $j_{1}, \ldots, j_{q}$ corresponding to those heat kernels which are followed by $I-H$. Thus,

$$
\begin{aligned}
S_{j_{0}, j_{1}, \ldots, j_{q}}= & t^{n} \int_{\Delta_{n}} \operatorname{Tr}\left(\gamma \ldots e^{-s_{j_{k}} t^{2} D^{2}}(I-H) \ldots\right. \\
& \left.H \ldots\left[D, a_{j_{h}}\right] e^{-s_{j_{h}} t^{2} D^{2}}(I-H) \ldots\right) d_{n} \sigma .
\end{aligned}
$$

In particular, the variables $s_{j}$ with $j \neq j_{0}, j_{1}, \ldots, j_{q}$, as well as $s_{j_{0}}$, do not appear in the integrand and all the remaining heat operators correspond to the invertible part of $D$. Identify $\Delta_{n}$ with the set

$$
\left\{\left(s_{0}, s_{1}, \ldots, s_{j_{0}+1}, s_{j_{0}-1}, \ldots, s_{n}\right) \in\left(\mathbb{R}^{+}\right)^{n} ; \sum_{j \neq j_{0}} s_{j} \leqq 1\right\},
$$

and then perform the substitution

$$
u_{k}=t^{2} s_{j_{k}} \text { for } k=1, \ldots, q \text { and } v_{j}=s_{j} \text { for } j \neq j_{0}, j_{1}, \ldots, j_{q} .
$$

Integrating first in the variables $v_{j}$, over the simplex

$$
\sum_{j \neq j_{0}, j_{1}, \ldots, j_{q}} v_{j} \leqq 1-t^{-2}\left(u_{1}+\cdots+u_{q}\right),
$$

and then over

$$
\left.\Delta_{q}(t)=\left\{u_{1}, \ldots, u_{q}\right) \in\left(\mathbb{R}^{+}\right)^{q} ; u_{1}+\cdots+u_{q} \leqq t^{2}\right\},
$$

one obtains

$$
\begin{aligned}
S_{j_{0}, j_{1}, \ldots, j_{q}}= & (n-q) !^{-1} t^{n-2 q} \int_{\Delta_{q(t)}}\left(1-t^{-2}\left(u_{1}+\cdots+u_{q}\right)\right)^{n-q} \\
& \times \operatorname{Tr}\left(\gamma \ldots e^{-u_{k} D^{2}}(I-H) \ldots H \ldots\left[D, a_{j_{h}}\right]\right. \\
& \left.\times e^{-u_{h} D^{2}}(I-H) \ldots\right) d_{n} \sigma
\end{aligned}
$$

When $t \rightarrow \infty$ the integral is dominated by

$$
\begin{gathered}
\int_{\left(\mathbb{R}^{+}\right)_{q}}\left|1+\left(u_{1}+\cdots+u_{q}\right)\right|^{n-q} \mid \operatorname{Tr}\left(\gamma \ldots e^{-u_{k} D^{2}}(I-H) \ldots\right. \\
\left.\ldots H \ldots\left[D, a_{j_{h}}\right] e^{-u_{h} D^{2}}(I-H) \ldots\right) \mid d u_{1} \ldots d u_{q}<\infty .
\end{gathered}
$$

On the other hand, the exponent $n-2 q$ is always $\leqq 0$, since $q \geqq[n / 2]$ (resp. $[n / 2]+1$ for $n$ odd). Moreover, the equality $n=2 q$ occurs only when $n$ is even, $q=n / 2$ and

$$
\begin{aligned}
S_{0,1,3, \ldots 2 q-1}= & q !^{-1} \int_{\Delta_{q(t)}}\left(1-t^{-2}\left(u_{1}+\ldots+u_{q}\right)\right)^{q} \\
& \times \operatorname{Tr}\left(\gamma a_{0} H\left[D, a_{1}\right] e^{-u_{1} D^{2}}(I-H)\left[D, a_{2}\right] H \ldots\right. \\
& \left.\ldots\left[D, a_{2 q-1}\right] e^{-u_{2 q-1} D^{2}}(I-H)\left[D, a_{2 q}\right] H\right) d u_{1} \ldots d u_{q}
\end{aligned}
$$


The limit for $t \rightarrow \infty$ of this term is

$$
\begin{aligned}
& q !^{-1} \int_{\left(\mathbb{R}^{+}\right)_{q}} \operatorname{Tr}\left(\gamma a_{0} H\left[D, a_{1}\right] e^{-u_{1} D^{2}}(I-H)\left[D, a_{2}\right] H \ldots\right. \\
&\left.\ldots\left[D, a_{2 q-1}\right] e^{-u_{2 q-1} D^{2}}(I-H)\left[D, a_{2 q}\right] H\right) d u_{1} \ldots d u_{q} \\
&=q !^{-1} \operatorname{Tr}\left(\gamma H a_{0} H\left[D, a_{1}\right] D^{-2}(I-H)\left[D, a_{2}\right] H\right. \\
&\left.\ldots\left[D, a_{2 q-1}\right] D^{-2}(I-H)\left[D, a_{2 q}\right] H\right) \\
&=q !^{-1} \operatorname{Tr}\left(\gamma H a_{0} H\left[D, a_{1}\right] D^{-1}(I-H) D^{-1}\left[D, a_{2}\right] H\right. \\
&\left.\quad \ldots\left[D, a_{2 q-1}\right] D^{-1}(I-H) D^{-1}\left[D, a_{2 q}\right] H\right) \\
&=q !^{-1} \operatorname{Tr}\left(\gamma H a_{0} H\left(-H a_{1}(I-H) a_{2} H\right) \ldots\left(-H a_{2 q-1}(I-H) a_{2 q} H\right)\right) \\
&=(-1)^{q} q !^{-1} \operatorname{Tr}\left(\gamma H a_{0} H\left(H a_{1} a_{2} H-H a_{1} H a_{2} H\right)\right. \\
&\left.\ldots\left(H a_{2 q-1} a_{2 q} H-H a_{2 q-1} H a_{2 q} H\right)\right) .
\end{aligned}
$$

2.3. The Transgressed Cocycle. In conjunction with Lemma 1(b), Lemma 2 enables us to define, for $n>p$, the "transgressed" cochain

$$
\mathrm{T}_{\phi} \mathrm{h}_{\infty}{ }^{n}(D)\left(a_{0}, \ldots, a_{n}\right)=\int_{0}^{\infty} \phi \mathrm{h}^{n}(s D, D)\left(a_{0}, \ldots, a_{n}\right) d s
$$

An easy calculation gives

$$
\begin{gathered}
\quad B \operatorname{Teh}_{\infty}{ }^{n+1}(D)\left(a_{0}, \ldots, a_{n}\right)=\tau^{n}{ }_{D}\left(a_{0}, \ldots, a_{n}\right)=(\text { see } 1.5) \\
=\sum_{\lambda} \operatorname{sgn}(\lambda) \int_{0}^{\infty} d t t^{n+1} \int_{\Delta_{n+1}} \operatorname{Tr}\left(\gamma D e^{-t_{1} t^{2} D^{2}}\left[D, a_{\lambda(0)}\right] e^{-\left(t_{2}-t_{1}\right) t^{2} D^{2}} \ldots\right. \\
\left.\ldots\left[D, a_{\lambda(n)}\right] e^{-\left(1-t_{n+1}\right) t^{2} D^{2}}\right) d t_{1} \ldots d t_{n+1},
\end{gathered}
$$

where $\lambda$ runs through the cyclic permutations of $\{0,1, \ldots, n\}$.

Theorem 1. Let $n>p-1, n=2 k$ or $2 k+1$, of the same parity as $(\mathbf{H}, D)$. Then

$$
\begin{aligned}
\operatorname{ch}_{\infty}{ }^{n}(D) \equiv & \sum_{0 \leqq j \leqq k}(-1) j !^{-1} \operatorname{Str}\left(\rho_{H}\left(a_{0}\right) \omega_{H}\left(a_{1}, a_{2}\right) \ldots \omega_{H}\left(a_{2 j-1}, a_{2 j}\right)\right. \\
+ & \sum_{\lambda} \operatorname{sgn}(\lambda) \int_{0}^{\infty} d t t^{n+1} \int_{\Delta_{n+1}} \operatorname{Tr}\left(\gamma D e^{-t_{1} t^{2} D^{2}}\left[D, a_{\lambda(0)}\right] e^{-\left(t_{2}-t_{1}\right) t^{2} D^{2}} \ldots\right. \\
\ldots & \left.\ldots\left[D, a_{\lambda(n)}\right] e^{-\left(1-t_{n+1}\right) t^{2} D^{2}}\right) d t_{1} \ldots d t_{n+1}
\end{aligned}
$$

in the even case, respectively

$$
\begin{array}{r}
\operatorname{ch}_{\infty}{ }^{n}(D) \equiv \sum_{\lambda} \operatorname{sgn}(\lambda) \int_{0}^{\infty} d t t^{n+1} \int_{\Delta_{n+1}} \operatorname{Tr}\left(D e^{-t_{1} t^{2} D^{2}}\left[D, a \lambda_{(0)}\right] e^{-\left(t_{2}-t_{1}\right) t^{2} D^{2}} \ldots\right. \\
\left.\ldots\left[D, a \lambda_{(n)}\right] e^{-\left(1-t_{n+1}\right) t^{2} D^{2}}\right) d t_{1} \ldots d t_{n+1},
\end{array}
$$

in the odd case, defines a cocycle in Tot ${ }_{+}(A)$ cohomologous to $\operatorname{ch}_{t}{ }^{n}(D), \forall t>0$, and therefore representing the same cohomology class in $H C^{n}(A)$. 
Proof. In view of the results in 2.2

$$
\operatorname{ch}_{\infty}{ }^{n}(D)=\lim _{t \rightarrow \infty} \operatorname{ch}_{t}{ }^{n}(D) .
$$

Now letting $\tau \rightarrow \infty$ in (2.2) one obtains

$$
\operatorname{ch}_{t}{ }^{n}(D)-\operatorname{ch}_{\infty}{ }^{n}(D)=(b+B) \sum_{k \geqq 0} \int_{t}^{\infty} \phi \mathrm{h}^{n-2 k-1}(s D, D) d s .
$$

Remark 1. If we make the assumption that $D$ is invertible (as in [CM1]), then

$$
\operatorname{ch}_{\infty}{ }^{n}(D)=\tau^{n}{ }_{D}, \quad \forall n>p-1,
$$

both in the even and in the odd case. In particular, this gives a direct proof to the fact that $\tau^{n}{ }_{D}$ is a cyclic cocycle. (The proof given in [CM1] relies on transgression in Quillen's superconnection formalism and the Loday-Quillen isomorphism [LQ].)

Remark 2. Still under the assumption that $D$ is invertible, one can give the following alternate interpretation to the above cocycle, which is closely related to the $\eta$-cochain of [Wu]. The total complex $\operatorname{Tot}_{+}(A)$ can be viewed as a subcomplex of the complex $\operatorname{Tot}_{\Pi}(A)$ of infinite cochains. The corresponding quotient complex $\operatorname{Qot}(A)=\left(C Q^{n}(A), \partial\right)$ can be described as follows:

$$
\begin{aligned}
C Q^{n}(A) & =\prod_{k \geqq 0} C^{n+2 k}(A), \\
\partial\left(\phi^{n}, \ldots, \phi^{n+2 k}, \ldots\right) & =\left(b \phi^{n}+B \phi^{n+2}, \ldots, b \phi^{n+2 k}+B \phi^{n+2 k+2}, \ldots\right) .
\end{aligned}
$$

Now the exact sequence

$$
0 \rightarrow \operatorname{Tot}_{+} *(A) \rightarrow \operatorname{Tot}_{\Pi 1}^{*}(A) \rightarrow \text { Qot }_{+}^{*+2}(A) \rightarrow 0,
$$

gives rise to a long exact cohomology sequence

$$
\ldots \rightarrow H C^{n-1}(A) \rightarrow H C_{\mathrm{inf}}^{n-1}(A) \rightarrow H Q^{n+1}(A) \rightarrow H C^{n}(A) \rightarrow \ldots,
$$

where $H Q^{*}(A)$ denotes the homology of Qot $(A)$. But the cohomology with infinite supports $H C_{\text {inf }}{ }^{n}(A)=0$, therefore the connecting homomorphism

$$
\delta: H Q^{n+1}(A) \rightarrow H C^{n}(A)
$$

is actually an isomorphism; moreover, it can be explicitly described as follows:

$$
\delta\left[\phi^{n+1}, \ldots, \phi^{n+2 k+1}, \ldots\right]=\left[0, \ldots, 0, B \phi^{n+1}\right] .
$$

Returning now to our cocycle, observe that, by letting $t \rightarrow \infty$ in formula (2.3), one gets for any $n>p-1$,

$$
b \mathrm{~T} \phi \mathrm{h}_{\infty}{ }^{n+2 k+1}(D)+B \mathrm{~T} \phi \mathrm{h}_{\infty}{ }^{n+2 k+3}(D)=-\mathrm{Ch}^{n+2 k+2}(\infty D)=0 .
$$

This shows that the cochain

$$
\mathrm{Q} \notin \mathrm{h}^{n}(D)=\left(\mathrm{T}_{\infty} \mathrm{h}^{n+1}(D), \ldots, \mathrm{T}_{\infty} \mathrm{h}^{n+1+2 k+1}(D), \ldots\right) \in C Q^{n+1}(A)
$$

is actually a cocycle. Moreover, from the above description of the connecting homomorphism, it follows that

$$
\left[\operatorname{ch}_{\infty}{ }^{n}(D)\right]=\delta^{-1}\left[\mathrm{Q} \phi \mathrm{h}^{n}(D)\right] .
$$




\section{Identification with the Chern Character}

3.1. Homotopy Invariance. In order to prove that the cohomology class of the completed transgressed cocycle coincides with the Chern character of [C1], we need to establish its invariance under special homotopies of the operator $D$.

We begin with some notation. Given an operator $V$ (possibly unbounded) on $\mathbf{H}$, and $A_{0}, \ldots, A_{n} \in \mathscr{L}(\mathbf{H})$, we set

$$
\begin{aligned}
\imath(V) & \left\langle A_{0}, \ldots, A_{n}\right\rangle_{D} \\
\quad & =\sum_{0 \leqq i \leqq n}(-1)^{[\mathrm{V}]\left(\left[A_{0}\right]+\left[A_{1}\right]+\ldots+\left[A_{i}\right]\right)}\left\langle A_{0}, \ldots, A_{i}, V, A_{i+1}, \ldots, A_{n}\right\rangle_{D}
\end{aligned}
$$

and then extend this definition by linearity. Note that, in particular,

$$
\phi \mathrm{h}^{n}(D, V)\left(a_{0}, \ldots, a_{n}\right)=\imath(V)\left\langle a_{0},\left[D, a_{1}\right], \ldots,\left[D, a_{n}\right]\right\rangle_{D} .
$$

We further define, for $V$ and $W$ as above,

$$
\begin{gathered}
\quad \mathbf{h}^{n}(D, V, W)\left(a_{0}, \ldots, a_{n}\right)=\imath(W) l(V)\left\langle a_{0},\left[D, a_{1}\right], \ldots,\left[D, a_{n}\right]\right\rangle_{D}, \\
\alpha^{n}(D, V, W)\left(a_{0}, \ldots, a_{n}\right) \\
\quad=\sum_{0 \leqq i \leqq n}(-1)^{(i-1)([V]+1)} l(W)\left\langle a_{0},\left[D, a_{1}\right], \ldots,\left[V, a_{i}\right], \ldots,\left[D, a_{n}\right]\right\rangle_{D} .
\end{gathered}
$$

Remark that

$$
\phi \mathrm{h}^{n}(D, V, W)+\phi \mathrm{h}^{n}(D, W, V)=0 .
$$

Lemma 3. With the above notation,

$$
\begin{aligned}
b \notin \mathrm{h}^{n-1}(D, V, W)+B \phi \mathrm{h}^{n+1}(D, V, W)= & \phi \mathrm{h}^{n}(D,[D, V], W)-\not \mathrm{h}^{n}(D,[D, W], V) \\
& -\alpha^{n}(D, V, W)+\alpha^{n}(D, W, V) .
\end{aligned}
$$

Proof. The verification of this equality uses the identities (1)-(4) of Lemma 2.2 in [GS] (or, equivalently, Propositions (IV.4)-(IV.6) of [JLO]). It is lengthy and tedious but rather straightforward, so we omit the details.

We now consider a one-parameter family $\left(\mathbf{H}, D_{\tau}\right)$ of bounded perturbations of a fixed $p$-summable, unbounded, Fredholm modules $(\mathbf{H}, D)$, of the form:

$D_{\tau}=D+\tau A$, with $A$ bounded, selfadjoint and also odd in the graded case .

Lemma 4. The p-summability property is stable under perturbations by bounded, selfadjoint operators.

Proof. Replace the function $e^{-x}$ in the proof of [GS, Theorem C] with the function $(1+x)^{-p / 2}$ and use the Mellin transform.

Lemma 5. With $D_{\tau}$ as above,

(a) $(d / d \tau) \phi \mathrm{h}^{n}\left(t D_{\tau}, D_{\tau}\right)=\not \mathrm{h}^{n}\left(t D_{\tau}, A\right)-\not \mathrm{h}^{n}\left(t D_{\tau},\left[t D_{\tau}, t A\right], D_{\tau}\right)+\alpha^{n}\left(t D_{\tau}, t A, D_{\tau}\right)$;

(b) $(d / d t) \phi \mathrm{h}^{n}\left(t D_{\tau}, t A\right)=\phi \mathrm{h}^{n}\left(t D_{\tau}, A\right)-\phi \mathrm{h}^{n}\left(t D_{\tau},\left[t D_{\tau}, D_{\tau}\right], t A\right)+\alpha^{n}\left(t D_{\tau}, D_{\tau}, t A\right)$;

(c) $(d / d t) \notin \mathrm{h}^{n}\left(t D_{\tau}, t A\right)-(d / d \tau) \phi \mathrm{h}^{n}\left(t D_{\tau}, D_{\tau}\right)=b \notin \mathrm{h}^{n-1}\left(t D_{\tau}, D_{\tau}, t A\right)$

$$
+B \phi \mathrm{h}^{n+1}\left(t D_{\tau}, D_{\tau}, t A\right) \text {. }
$$


Proof. The identities (a) and (b) can be easily checked by using Duhamel's formula for the derivative of the heat operator, while (c) follows by subtracting them and applying Lemma 3.

Proposition 3. Let $\left(\mathbf{H}, D_{\tau}\right)$ be a one-parameter family of the form (3.1). Then for any $n>p-1, n$ of the same parity as $(\mathbf{H}, D)$, one has

$$
\begin{aligned}
\operatorname{ch}_{t}{ }^{n}\left(D_{0}\right)-\operatorname{ch}_{t}{ }^{n}\left(D_{1}\right)= & (b+B) \int_{0}^{1}\left(\sum_{k \geqq 0} \phi \mathrm{h}^{n-2 k-1}\left(t D_{\tau}, t A\right)\right. \\
& \left.+b \int_{0}^{t} \phi \mathrm{h}^{n}\left(s D_{\tau}, D_{\tau}, s A\right) d s\right) d \tau
\end{aligned}
$$

Proof. The transgression formula for the JLO cocycle with respect to the parameter $\tau$ gives, for any $t>0$,

$$
-(d / d \tau) \mathrm{Ch}^{l}\left(t D_{\tau}\right)=b \notin \mathrm{h}^{l-1}\left(t D_{\tau}, t A\right)+B \notin \mathrm{h}^{l+1}\left(t D_{\tau}, t A\right) .
$$

Thus,

$$
\begin{aligned}
-(d / d \tau) \mathrm{ch}_{t}{ }^{n}\left(D_{\tau}\right)= & -\sum_{k \geqq 0}(d / d \tau) \mathrm{Ch}^{n-2 k}\left(t D_{\tau}\right)-B(d / d \tau) T \phi \mathrm{h}_{t}{ }^{n+1}\left(D_{\tau}\right) \\
= & (b+B) \sum_{k \geqq 0} \phi \mathrm{h}^{n-2 k-1}\left(t D_{\tau}, t A\right) \\
& +B\left(\phi \mathrm{h}^{n+1}\left(t D_{\tau}, t A\right)-(d / d \tau) \mathrm{T}_{\phi} \mathrm{h}_{t}{ }^{n+1}\left(D_{\tau}\right)\right) .
\end{aligned}
$$

On the other hand, integrating the identity (c) of Lemma 5 from 0 to $t$ one obtains

$$
\begin{aligned}
\phi \mathrm{h}^{n+1}\left(t D_{\tau}, t A\right)-(d / d \tau) \mathrm{T} \phi \mathrm{h}_{t}{ }^{n+1}\left(D_{\tau}\right)= & b \int_{0}^{t} \phi \mathrm{h}^{n}\left(s D_{\tau}, D_{\tau}, s A\right) d s \\
& +B \int_{0}^{t} \phi \mathrm{h}^{n+2}\left(s D_{\tau}, D_{\tau}, s A\right) d s .
\end{aligned}
$$

Here we have taken into account that, by (the proof of) Lemma 1 , when $t \rightarrow 0^{+}$,

$$
\left\|\not \mathrm{h}^{n+1}\left(t D_{\tau}, t A\right)\right\|=t\left\|\phi \mathrm{h}^{n+1}\left(t D_{\tau}, A\right)\right\|=O\left(t^{n+2-p}\right),
$$

whereas

$$
\begin{aligned}
\left\|\phi \mathrm{h}^{n+1}\left(t D_{\tau}, D_{\tau}\right)\right\| & =O\left(t^{n-p}\right) \\
\left\|\phi \mathrm{h}^{n}\left(t D_{\tau}, D_{\tau}, t A\right)\right\| & =t\left\|\phi \mathrm{h}^{n}\left(t D_{\tau}, D_{\tau}, A\right)\right\|=O\left(t^{n-p}\right)
\end{aligned}
$$

Therefore,

$$
B\left(\phi \mathrm{h}^{n+1}\left(t D_{\tau}, t A\right)-(d / d \tau) \mathrm{T} \phi \mathrm{h}_{t}{ }^{n+1}\left(D_{\tau}\right)\right)=(b+B) b \int_{0}^{t} \phi \mathrm{h}^{n}\left(s D_{\tau}, D_{\tau}, s A\right) d s .
$$

It follows that

$$
-(d / d \tau) \operatorname{ch}_{t}{ }^{n}\left(D_{\tau}\right)=(b+B)\left(\sum_{k \geqq 0} \phi \mathrm{h}^{n-2 k-1}\left(t D_{\tau}, t A\right)+b \int_{0}^{t} \phi \mathrm{h}^{n}\left(s D_{\tau}, D_{\tau}, s A\right) d s\right),
$$

which completes the proof. 
Remark 3. The assumption (3.1), which suffices for the purposes of this paper, can be considerably relaxed. Along the same lines, but with additional technical effort, one can establish the homotopy invariance under much more general conditions, e.g. those discussed in [EFJL].

3.2. The Chern Character Theorem. We are now ready to prove the main result of this article.

Theorem 2. Let $n>p-1, n=2 k$ or $2 k+1$, of the same parity as $(\mathbf{H}, D)$. Then the cocycle $\operatorname{ch}_{\infty}{ }^{n}(D)$ represents the Chern character $\operatorname{ch}^{n}(\mathbf{H}, D) \in H C^{n}(A)$.

Proof. As explained in 1.5, it suffices to treat the even case. Let $\left(\mathbf{H} \otimes_{\mathrm{gr}} \mathbf{h}, D_{m}\right)$ be the "Hamiltonian amplification" of $(\mathbf{H}, D)$ (see 1.5). We need to show that, for $m \neq 0$,

$$
\left[\operatorname{ch}_{\infty}{ }^{n}(D)\right]=\operatorname{ch}^{n}\left(\mathbf{H} \otimes_{\mathrm{gr}} \mathbf{h}, D_{m}\right) \in H C^{n}(A) .
$$

Since $D_{m}$ is invertible for $m \neq 0$, by Remark 1 and [CM1],

$$
\operatorname{ch}^{n}\left(\mathbf{H} \otimes_{\mathrm{gr}} \mathbf{h}, D_{m}\right)=\left[\operatorname{ch}_{\infty}{ }^{n}\left(D_{m}\right)\right] .
$$

On the other hand, since

$$
D_{m}=D \otimes_{\mathrm{gr}} I+m I \otimes_{\mathrm{gr}} F,
$$

Proposition 3 is applicable and ensures that

$$
\left[\mathrm{ch}_{\infty}{ }^{n}\left(D_{m}\right)\right]=\left[\mathrm{ch}_{\infty}{ }^{n}\left(D_{0}\right)\right] .
$$

Finally, a simple inspection of the definitions shows that

$$
\operatorname{ch}_{\infty}{ }^{n}\left(D_{0}\right)=\operatorname{ch}_{\infty}{ }^{n}(D) \text {. }
$$

3.3. The Index Pairing. Let us first recall the construction of the Chern character map in cyclic homology. Given an idempotent matrix $e=e^{2} \in M_{r}(A)$, the formal sum

$$
\operatorname{ch}(e)=\operatorname{tr}_{0}(e)+\sum_{k \geqq 1}(-1)^{k}(2 k) ! / k !\left(\operatorname{tr}_{2 k+1}\left(e^{\otimes 2 k+1}\right)-2^{-1} \operatorname{tr}_{2 k+1}\left(1 \otimes e^{\otimes 2 k}\right)\right),
$$

where $\operatorname{tr}_{k}: M_{r}(A)^{\otimes k} \rightarrow A^{\otimes k}$ is the generalized trace map, defines a cycle in the periodic $(b, B)$-bicomplex, and its homology class $\operatorname{ch}(\mathbf{e}) \in H C_{\mathrm{ev}}^{\mathrm{per}}(A)$ depends only on the K-theory class $\mathbf{e} \in K_{0}(A)$ of $e$ (cf. [HJ]). Furthermore, there is a natural pairing

$$
\langle,\rangle: H C^{*}{ }_{\text {per }}(A) \times H C_{*}{ }^{\text {per }}(A) \rightarrow \mathbb{C} .
$$

Let now $(\mathbf{H}, D)$ be an even p-summable unbounded Fredholm module over $A$. Let $\mathbf{e} \in K_{0}(A), e=e^{2} \in M_{r}(A)$ and form the Fredholm operator

$$
D_{e}^{+}=e\left(D^{+} \otimes I\right) e: e\left(\mathbf{H}^{+} \otimes \mathbb{C}^{r}\right) \rightarrow e\left(\mathbf{H}^{-} \otimes \mathbb{C}^{r}\right) .
$$

According to the index theorem of [C1],

$$
\operatorname{Index} D_{e}^{+}=\langle\mathbf{c h}(\mathbf{H}, D), \boldsymbol{c h}(e)\rangle, \quad \forall n=2 k>p-1 .
$$


This also follows, along the lines of [C2; Sect. 7], from the preceding results. Indeed, without loss of generality but passing to a larger algebra, one may assume $e$ to be self-adjoint. Then, by means of the homotopy

$$
D_{\tau}=D+\tau(2 e-1)[D, e], \quad \tau \in[0,1],
$$

one reduces the general case to the situation when $D$ and $e$ commute, in which case the above formula becomes obvious.

As a consequence of Theorems 1 and 2, and assuming for simplicity $r=1,(3.3)$ takes the form:

$$
\begin{aligned}
\operatorname{Index} D_{e}^{+}= & \operatorname{Str} \rho_{H}(e)+\sum_{1 \leqq j \leqq k}(2 j) ! /(j !)^{2} \operatorname{Str}\left(\left(\rho_{H}(e-1 / 2) \omega_{H}(e, e)^{j}\right)\right. \\
& +(-1)^{k}(2 k) ! / k ! \sum_{\lambda} \operatorname{sgn}(\lambda) \int_{0}^{\infty} d t t^{n+1} \\
& \times \int_{\Delta_{n+1}} \operatorname{Tr}\left(\gamma D e^{-t_{1} t^{2} D^{2}}[D, e] e^{-\left(t_{2}-t_{1}\right) t^{2} D^{2}} \ldots\right. \\
& \left.\ldots[D, e] e^{-\left(1-t_{n+1}\right) t^{2} D^{2}}\right) d t_{1} \ldots d t_{n+1}, \quad \forall n=2 k>p-1 .
\end{aligned}
$$

\section{Infinite Temperature Limit}

4.1. Large Temperature Expansion. We shall now make the following assumption:

For each $l \geqq 0$, the vector valued functions $\phi(t)=\mathrm{Ch}^{l}(t D)$ or $\mathrm{ch}^{l}(t D, D)$ can be expressed on $(0, T], T>0$, in the form

$$
\begin{aligned}
\phi(t)=\psi(t) & +\sum_{1 \leqq k \leqq K}\left[\alpha_{k}+\sum_{1 \leqq j \leqq J} \alpha_{k j} \log ^{j} t\right] t^{-a_{k}} \\
& +\sum_{1 \leqq k \leqq K}\left[\beta_{k}+\sum_{1 \leqq j \leqq J} \beta_{k j} t \log ^{j} t\right] t^{-k}
\end{aligned}
$$

with $\psi(t)$ continuous on $[0, T], a_{k} \neq 0,1,2,3, \ldots, \psi(t)$ and all the coefficients in $C^{l}(A)$.

Let us recall that for such a function $\phi(t)$ one defines its finite part at $0^{+}$as $\mathrm{Pf}_{t=0_{+}} \phi=\psi\left(0^{+}\right)$. Similarly, one defines the finite part integral $\operatorname{Pf} \int_{0}^{T} \phi(t) d t$ by removing the divergence at $0^{+}$.

Thus, in the presence of the above hypothesis, we can extend the definition of the cochain $\operatorname{T}_{\phi} h^{n}(D)$ to all $n \geqq 0$, simply by replacing the ordinary integral with the finite part integral:

$$
\operatorname{PfT}_{\phi} h_{t}{ }^{n}(D)\left(a_{0}, \ldots, a_{n}\right)=\operatorname{Pf} \int_{0}^{t} \phi \mathrm{h}^{n}(s D, D)\left(a_{0}, \ldots, a_{n}\right) d s .
$$

Similarly, replacing the limit at $0^{+}$by the finite part at $0^{+}$, we define

$$
\operatorname{PfCh}^{n}(D)=\operatorname{Pf}_{t=0+} \mathrm{Ch}^{n}(t D), \quad \forall n \geqq 0 .
$$


Note that, by Lemma 1(a), one has

$$
\operatorname{PfCh}^{n}(D)=0, \quad \forall n>p .
$$

Proposition 4. For any $n>p-1, n$ of the same parity as $(\mathbf{H}, \mathbf{D})$, the formula

$$
\operatorname{Pfch}^{n}(D)=\sum_{k \geqq 0} \operatorname{PfCh}^{n-2 k}(D),
$$

defines a cocycle cohomologous to $\mathrm{ch}_{t}{ }^{n}(D), \forall 0<t \leqq \infty$.

Proof. Indeed, by integrating (1.2) from 0 to $t$, one obtains:

$$
\operatorname{PfCh}^{l}(D)-\operatorname{Ch}^{l}(t D)=b\left(\operatorname{PfT}_{\phi h_{t}^{l-1}}^{l}(D)\right)+B\left(\operatorname{PfT}_{\phi} h_{t}^{l+1}(D)\right), \quad \forall l \geqq 0 .
$$

Therefore,

$$
\begin{aligned}
\operatorname{Pfch}^{n}(D)-\operatorname{ch}_{t}{ }^{n}(D) & =\sum_{k \geqq 0}\left(\operatorname{PfCh}^{n-2 k}(D)-\mathrm{Ch}^{n-2 k}(t D)\right)-B \operatorname{Teh}_{t}{ }^{n+1}(D) \\
& =(b+B) \sum_{k \geqq 0} \operatorname{PfT}_{t} h_{t}{ }^{n-2 k-1}(D) . \square
\end{aligned}
$$

In particular, the index formula (3.4) takes the "finite" form:

$$
\begin{aligned}
\text { Index } D_{e}^{+}= & \operatorname{Pf}_{t=0+} \operatorname{Tr}\left(\gamma e e^{-t^{2} D^{2}}\right) \\
& +\sum_{1 \leqq j \leqq k}(-1)^{j}(2 j) ! / j ! \mathrm{Pf}_{t=0_{+}} \mathrm{Ch}^{2 j}(t D)(e-1 / 2, e, \ldots, e) . \\
& \forall 2 k>p-1 .
\end{aligned}
$$

Remark 4. The assumption (4.1) is satisfied when $D$ is the Dirac operator $D$ on a closed spin-manifold $M$ (or even a generalized Dirac operator on a closed manifold) - see [BF ] and [Wu]. Moreover, $\operatorname{Pfch}^{n}(\boxplus)$ can be explicitly computed by means of Getzler's asymptotic calculus (cf. [Ge], [CM2; §3]) and gives precisely the current representing the homology class $\mathrm{ch}_{*}(\bigoplus)$. Indeed, one has

$$
\begin{gathered}
\operatorname{PfCh}^{2 k}(\boxplus)\left(a_{0}, \ldots, a_{2 k}\right)=c_{k} \int_{M} \hat{A}(M) \wedge a_{0} \wedge d a_{1} \wedge \ldots \wedge d a_{2 k}, \\
\forall a_{0}, \ldots, a_{2 k} \in C^{\infty}(M),
\end{gathered}
$$

where $c_{k}$ are certain universal constants and

$$
\hat{A}(M)=\operatorname{det}^{1 / 2}\left(\frac{R / 2}{\sinh R / 2}\right)
$$

is the $\hat{A}$-genus form of the manifold $M$ with respect to the Riemannian curvature $R$.

4.2. A Non-commutative Example. Let $\mathbf{T}^{0}\left(\mathbb{S}^{1}\right)$ be the algebra of pseudodifferential Toeplitz operators on the circle. We recall that a Toeplitz operator (with continuous symbol) on the circle is an operator of the form $T_{f}=P M_{f} P$, where $M_{f}$ is the multiplication by $f \in C\left(\mathbb{S}^{1}\right)$ on $L^{2}\left(\mathbb{S}^{1}\right)$ and $P$ is the orthogonal projection onto the Hardy space $H^{2}\left(\mathbb{S}^{1}\right)$. The Toeplitz operators generate a $C^{*}$-subalgebra $\mathbf{T}\left(\mathbb{S}^{1}\right)$ of $\mathscr{L}\left(H^{2}\left(\mathbb{S}^{1}\right)\right)$. Thus,

$$
\mathbf{T}^{0}\left(\mathbb{S}^{1}\right)=\mathbf{T}\left(\mathbb{S}^{1}\right) \cap \Psi^{0}\left(\mathbb{S}^{1}\right)
$$


where $\Psi^{k}\left(\mathbb{S}^{1}\right)$ denotes the algebra of (classical) pseudodifferential operators on the circle of order equal or less than $k$. The algebra $\mathbf{T}^{0}\left(\mathbb{S}^{1}\right)$ is included into an extension

$$
0 \rightarrow \Psi^{-\infty}\left(\mathbb{S}^{1}\right) \rightarrow \mathbf{T}^{0}\left(\mathbb{S}^{1}\right) \rightarrow C S^{0}+\left(\mathbb{S}^{1}\right) \rightarrow 0
$$

where $C S^{0}+\left(\mathbb{S}^{1}\right)=\left\{\alpha=\sum_{j \geqq 0} \alpha_{j} \xi^{-j} ; \alpha_{j} \in C^{\infty}\left(\mathbb{S}^{1}\right), \xi>0\right\}$ is the "positive half" of the complete symbol algebra $C S^{0}\left(\mathbb{S}^{1}\right)$.

The characters $\left\{\varepsilon_{k} ; \varepsilon_{k}(x)=e^{i k x}, k \in \mathbb{Z}^{+}\right\}$form an orthonormal basis of $H^{2}\left(\mathbb{S}^{1}\right)$, identifying it to $l^{2}\left(\mathbb{Z}^{1}\right)$. Let $S$ denote the unilateral shift $S \varepsilon_{k}=\varepsilon_{k+1}$ and let $N$ be the "number operator" $N \varepsilon_{k}=k \varepsilon_{k}$. For each $n \in \mathbb{N}$, we define an even unbounded Fredholm module over $\mathbf{T}^{0}\left(\mathbb{S}^{1}\right)$, of index $n$, as follows: $\mathbf{H}=\mathbf{H}^{+} \oplus \mathbf{H}^{-}$, with $\mathbf{H}^{ \pm}=l^{2}\left(\mathbb{Z}^{+}\right), a \in \mathbf{T}^{0}\left(\mathbb{S}^{1}\right)$ acts as $a^{\sim}=a \oplus a$ and

$$
D_{n}=\left[\begin{array}{ll}
0 & D_{n}^{-} \\
D_{n}^{+} & 0
\end{array}\right],
$$

where $D_{n}{ }^{-}=N^{1 / 2} S^{n}, D_{n}{ }^{+}=\left(D_{n}^{-}\right)^{*}$. In particular $D_{1}{ }^{+}\left(\right.$resp. $\left.D_{1}^{-}\right)$is the "annihilation" (resp. "creation") operator of the simple harmonic oscillator in one dimension.

Clearly $D_{n}{ }^{ \pm} \in \Psi^{1 / 2}\left(\mathbb{S}^{1}\right)$, in particular $\left(\mathbf{H}, D_{n}\right)$ is $(2+\varepsilon)$-summable. Moreover, from the standard symbolic calculus it follows that $\left(\mathbf{H}, D_{n}\right)$ satisfies the assumption (4.1) and we proceed to compute its "local" Chern character.

One has

$$
D_{n}{ }^{2}=N\left[\begin{array}{ll}
I-P_{n} & 0 \\
0 & I
\end{array}\right]+n\left[\begin{array}{ll}
0 & 0 \\
0 & I
\end{array}\right]
$$

where $P_{n}$ denotes the orthogonal projection onto $\sum_{0 \leqq k \leqq n-1} \mathbb{C} \varepsilon_{k}$. Hence

$$
\exp \left(-t D_{n}{ }^{2}\right)=\left[\begin{array}{ll}
P_{n} & 0 \\
0 & 0
\end{array}\right]+e^{-t N}\left[\begin{array}{ll}
I-P_{n} & 0 \\
0 & I
\end{array}\right]+e^{-t n} e^{-t N}\left[\begin{array}{ll}
0 & 0 \\
0 & I
\end{array}\right] .
$$

By an easy calculation one gets, for any $a \in \mathbf{T}^{0}\left(\mathbb{S}^{1}\right)$,

$$
\operatorname{Tr}\left(\gamma a^{\sim} \exp \left(-t D_{n}{ }^{2}\right)\right)=\operatorname{Tr}\left(a P_{n}\left(I-e^{-t N}\right)\right)+\left(1-e^{-t n}\right) \operatorname{Tr}\left(a e^{-t N}\right),
$$

which implies

$$
\mathrm{Pf}_{t=0_{+}} \operatorname{Tr}\left(\gamma a^{\sim} \exp \left(-t D_{n}^{2}\right)\right)=n \mathrm{Pf}_{t=0_{+}} t \operatorname{Tr}\left(a e^{-t N}\right) .
$$

In turn, this can be computed by means of an appropriate zeta-function. Indeed, let us note that

$$
\begin{aligned}
\mathrm{Pf}_{t=0_{+}} t \operatorname{Tr}\left(a e^{-t N}\right) & =\mathrm{Pf}_{t=0_{+}} t \operatorname{Tr}\left(a \exp \left(-t\left(N+P_{1}\right)\right)\right) \\
& =\mathrm{Pf}_{t=0_{+}} t \operatorname{Tr}(a \exp (-t A)),
\end{aligned}
$$

where $A=\left(\Delta+P_{1}\right)^{1 / 2}=\Delta^{1 / 2}+P_{1}$, with $\Delta=-d^{2} / d x^{2}=$ the Laplacian of the circle. Denoting by $\zeta(A+u a, s)$ the zeta function of the first-order elliptic operator $A+u a$, one has

$$
\operatorname{Pf}_{t=0+} t \operatorname{Tr}\left(a e^{-t N}\right)=-d /\left.d u\right|_{u=0} \zeta(A+u a, 0) .
$$


Following Seeley's recipe [S; p. 290, formula (II)], the value $\zeta(A+u a, 0)$ can be explicitly computed from the complete symbol of $A+u a$,

$$
\sigma(A+u a)(x, \xi)=|\xi|+u \sigma(a)(x, \xi)=|\xi|+u \sum_{j \geqq 0} \sigma_{-j}(a)(x, \xi)
$$

as follows:

$$
\zeta(A+u a, 0)=-u / 2 \pi \int_{0}^{2 \pi} \sigma_{0}(a)(x) d x
$$

Therefore,

$$
\operatorname{Pf}_{t=0_{+}} t \operatorname{Tr}\left(a e^{-t N}\right)=1 / 2 \pi \int_{0}^{2 \pi} \sigma_{0}(a)(x) d x,
$$

which shows that

$$
\mathrm{Pf}_{t=0_{+}} \mathrm{Ch}^{0}\left(D_{n}\right)(a)=n / 2 \pi \int_{0}^{2 \pi} \sigma_{0}(a)(x) d x, \quad \forall a \in \mathbf{T}^{0}\left(\mathbb{S}^{1}\right) .
$$

Because the right-hand side is a trace, one has

$$
(b+B) \operatorname{PfCh}^{0}\left(D_{n}\right)=b \operatorname{PfCh}^{0}\left(D_{n}\right)=0,
$$

so $\operatorname{PfCh}^{0}\left(D_{n}\right)$ represents a class in $H C^{0}\left(\mathbf{T}^{0}\left(\mathbb{S}^{1}\right)\right)$.

At this point let us pause to note that, according to Wodzicki [Wo],

$$
H C^{2 k}\left(\mathbf{T}^{0}\left(\mathbb{S}^{1}\right)\right) \cong H C^{2 k}\left(C S^{0}+\left(\mathbb{S}^{1}\right)\right), \quad \forall k \geqq 0
$$

and moreover there are canonical isomorphisms

$$
\begin{aligned}
H C^{0}\left(\mathbf{T}^{0}\left(\mathbb{S}^{1}\right)\right) & \cong \mathscr{E}_{0}\left(\mathbb{S}^{1}\right) \oplus \mathscr{E}_{1}\left(\mathbb{S}^{1}\right), \\
H C^{2 k}\left(\mathbf{T}^{0}\left(\mathbb{S}^{1}\right)\right) & \cong H_{0}{ }^{\mathrm{dR}}\left(\mathbb{S}^{1}\right), H C^{2 k-1}\left(\mathbf{T}^{0}\left(\mathbb{S}^{1}\right)\right)=0, \quad \forall k \geqq 1,
\end{aligned}
$$

where $\mathscr{E}_{q}\left(\mathbb{S}^{1}\right)=$ the space of $q$-currents on $\mathbb{S}^{1}$. In addition, the periodicity operator

$$
S: H C^{0}\left(\mathbf{T}^{0}\left(\mathbb{S}^{1}\right)\right) \cong \mathscr{E}_{0}\left(\mathbb{S}^{1}\right) \oplus \mathscr{E}_{1}\left(\mathbb{S}^{1}\right) \rightarrow H C^{2}\left(\mathbf{T}^{0}\left(\mathbb{S}^{1}\right)\right) \cong H_{0}{ }^{\mathrm{dR}}\left(\mathbb{S}^{1}\right)
$$

is the operator sending any 0 -current to its homology class and any 1-current to 0 .

Under the above isomorphism, $\left[\mathrm{PfCh}^{0}\left(D_{1}\right)\right] \in H C^{0}\left(\mathbf{T}^{0}\left(\mathbb{S}^{1}\right)\right)$ identifies to the 0 -current of integration over $\mathbb{S}^{1}$. It follows that $S\left[\operatorname{PfCh}^{0}\left(D_{n}\right)\right] \in H C^{2}\left(\mathbf{T}^{0}\left(\mathbb{S}^{1}\right)\right)$ identifies to $n$ times the canonical generator of $H_{0}{ }^{\mathrm{dR}}\left(\mathbb{S}^{1}\right)$.

On the other hand

$$
\operatorname{PfCh}^{2}\left(D_{n}\right)=\operatorname{Pfch}^{2}\left(D_{n}\right)-\operatorname{PfCh}^{0}\left(D_{n}\right),
$$

is also $(b+B)$-closed and hence represents a class $\left[\operatorname{PfCh}^{2}\left(D_{n}\right)\right] \in H C^{2}\left(\mathbf{T}^{0}\left(\mathbb{S}^{1}\right)\right)$. But since $H C^{2}\left(\mathbf{T}^{0}\left(\mathbb{S}^{1}\right)\right)$ is one-dimensional, it follows that

$$
\left[\operatorname{PfCh}^{2}\left(D_{n}\right)\right]=c S\left[\operatorname{PfCh}^{0}\left(D_{n}\right)\right],
$$

for some constant $c$. Furthermore, the index pairing with an idempotent $e \in M_{r}\left(\mathbf{T}^{0}\left(\mathbb{S}^{1}\right)\right)$ reads as follows:

$$
\operatorname{Index}\left(D_{n}{ }^{+}\right)_{e}=(1+c) n / 2 \pi \int_{0}^{2 \pi} \operatorname{Tr} \sigma_{0}(e)(x) d x .
$$


Taking $e=I$, one sees that $c$ must be 0 .

In conclusion, $\operatorname{ch}^{2}\left(\mathbf{H}, D_{n}\right) \in H C^{2}\left(\mathbf{T}^{0}\left(\mathbb{S}^{1}\right)\right) \cong H_{0}{ }^{\mathrm{dR}}\left(\mathbb{S}^{1}\right)$ identifies to the $n^{\text {th }}$ multiple of the canonical generator of $H_{0}\left(\mathbb{S}^{1}\right)$.

\section{References}

[BGV] Berline, N., Getzler, E., Vergne, M.: Heat Kernels and Dirac Operators. Berlin Heidelberg New-York: Springer-Verlag 1992

[BF] Block, J., Fox, J.: Asymptotic Pseudodifferential Operators and Index Theory. Contemp. Math. 105, 1-32 (1990)

[C1] Connes, A.: Non-commutative differential geometry. Publ. Math. IHES 62, 41-144 (1985)

[C2] Connes, A.: Entire cyclic cohomology of Banach algebras and characters of $\vartheta-$ summable Fredholm modules. $K$-theory 1, 519-548 (1988)

[C3] Connes, A.: On the Chern character of $\theta$-summable Fredholm modules. Commun. Math. Phys. 139, 171-181 (1991)

[CM1] Connes, A., Moscovici, H.: Transgression du caractère de Chern et cohomologie cyclique. C.R. Acad. Sci. Paris, Serie I 303, 913-918 (1986)

[CM2] Connes, A., Moscovici, H.: Cyclic cohomology, the Novikov conjecture and hyperbolic groups. Topology 29, 345-388 (1990)

[EFJL] Ernst, K., Feng, P., Jaffe, A., Lesniewski, A.: Quantum K-theory. II. Homotopy invariance of the Chern character. J. Funct. Anal. 90, 355-368 (1990)

[Ge] Getzler, E.: Pseudodifferential operators on supermanifolds and the Atiyah-Singer index theorem. Commun. Math. Phys. 92, 163-178 (1983)

[GS] Getzler, E., Szenes, A.: On the Chern character of a theta-summable Fredholm module. J. Funct. Anal. 84, 343-357 (1989)

[JLO] Jaffe, A., Lesniewski, A., Osterwalder, K.: Quantum K-theory. I. The Chern character. Commun. Math. Phys. 118, 1-14 (1988)

[HJ] Hood, C.E., Jones, J.D.: Some algebraic properties of cyclic homology groups. Ktheory 1, 361-384 (1987)

[LQ] Loday, J.-L., Quillen, D.G.: Cyclic homology and the Lie algebra homology of matrices. Comm. Mat. Helv. 59, 565-591 (1984)

[Q] Quillen, D.: Algebra cochains and cyclic cohomology. Publ. Math. IHES 68, 139-174 (1989)

[S] Seeley, R.T.: Complex Powers of an Elliptic Operator. Proc. Symp. Pure Math. 10, 288-307

[Wo] Wodzicki, M.: Cyclic homology of pseudodifferential operators and noncommutative Euler class. C.R. Acad. Sc. Paris, Serie I 306, 321-325 (1988)

$[\mathrm{Wu}] \mathrm{Wu}, \mathrm{F}$.: The Chern-Connes character for Dirac operators on manifolds with boundary. Preprint 1992 